\title{
Treatment Outcomes with Nitazoxanide in Immunocompetent Adults Naive Patients with Cryptosporidiosis; Do We Need Combination Therapy with Paromomycin or Azithromycin?
}

\section{Sajjad $\mathrm{Ali}^{1^{*}}$ and Sunil Kumar ${ }^{2}$}

${ }^{1}$ Chairman Infection Prevention and Control Department, Department of Internal Medicine/Infectious Diseases, Sultan Bin AbdulAziz Humanitarian City Hospital, Riyadh, Saudi Arabia

${ }^{2}$ Department of Infectious Diseases, Sindh Institute of Urology and Transplantation, Karachi, Pakistan

*Corresponding author: Sajjad Ali, Chairman Infection Prevention and Control Department, Department of Internal Medicine/Infectious Diseases, Sultan Bin AbdulAziz Humanitarian City Hospital, Riyadh, Saudi Arabia, Tel: 00966115620000; E-mail: sajjad_siut@yahoo.com

Received date: September 16, 2015, Accepted date: October 16, 2015, Published date: October 19, 2015

Copyright: @ 2015 Sajjad, et al. This is an open-access article distributed under the terms of the Creative Commons Attribution License, which permits unrestricted use, distribution and reproduction in any medium, provided the original author and source are credited.

\section{Abstract}

Introduction: Human cryptosporidiosis is caused by infection with Cryptosporidium. Nitazoxanide has shown activity against cryptosporidium. The objective of this study is to see treatment outcomes with 7 days of nitazoxanide in immunocompetent adult patients diagnosed with cryptosporidiosis and to consider combination therapy which includes nitazoxanide with paromomycin or azithromycin?

Study Design: This cross sectional study was conducted at Sindh Institute of Urology and Transplantation, Karachi Pakistan. Patients were not enrolled with prior diagnosis of cryptosporidiosis and/or had taken Nitazoxanide, Paromomycin or Azithromycin in last 4 weeks prior their diagnosis. Also patients were excluded if they were diagnosed as case of HIVIAIDS, history of solid organ transplantation, any malignancy or were taking steroids and immunosuppressant drugs.

Results: A total of 58 patients who had cryptosporidiosis were included in this study. $31(53.4 \%)$ were males and $27(46.6 \%)$ females. The mean age was 33.4 years with standard deviation \pm 9.2 . No statistical significance was seen in clinical presentation of cryptosporidiosis in both male and female genders. All $58(100 \%)$ reported resolution of diarrhea after 7 days of nitazoxanide treatment. However, at 6 weeks follow up, $40(70.1 \%)$ patients had recurrence of diarrhea whereas only $17(29.9 \%)$ had no further episode of diarrhea.

Conclusion: Nitazoxanide is a new nitrothiazole compound with broadspectrum activity against numerous intestinal protozoa and helminths and have very good bio-safety profile. All 58 patients after 7 days of treatment with nitazoxanide showed good clinical response after short term. But in long term patients reported a high recurrence in 6 weeks period time. There is need of combination therapy which includes nitazoxanide with paromomycin or azithromycin in high recurrence/relapse patients.

Keywords: Diarrhea; Paromomycin; Azithromycin

\section{Introduction}

Human cryptosporidiosis is caused by infection with . Ten different species have been recognized but two most common species include Cryptosporidium parvum and Cryptosporidium hominis [1]. This is an intracellular parasite and capable of completing all stages of its development asexual and sexual within a single host [2]. Probably this is could be reason for high recurrence or relapse. The common mode of transmission is via the faeco-oral route, from animal to human, human to human, as well as by ingestion of contaminated water or food ingestion. Unpasteurized milk products have been associated with Cryptosporidium infections, and consumption of such products has been identified as a risk factor for sporadic cases of infection [3].

Cryptosporidial infection accounts for up to $22 \%$ cases of diarrhea in immunocompetent persons in developed countries and up to $41 \%$ of cases of diarrhea in immunocompetent persons in developing countries [2]. Cryptosporidium commonly infest small intestine and primarily causing diarrhea. After 7 to 10 days of incubation period, immunocompetent patient presents mostly with self-limiting watery diarrhea of short duration often greater than 5 or 6 episodes per day associated with crampy abdominal pain, nausea, vomiting, dehydration and low grade fever. Non-immunocompromised patients usually get early recovery in 3 to 5 days [4].

Nitazoxanide is a nitrothiazolyl-salicylamide derivative which has shown activity against cryptosporidium and is effective in its treatment [5]. It was first described in 1975 by Jean Francois Rossignol. Other enteric protozoan infections caused by Giardia intestinalis, Entamoeba histolytica, Blastocystis hominis and Isospora belli can also be effectively treated with nitazoxanide [6]. Currently nitazoxanide is not recommended for empirical use in acute diarrhea; however there are some studies have shown results that are statistically significant [7].

The objective of this study is to see treatment outcomes with 7 days of nitazoxanide in immunocompetent adult patients diagnosed with cryptosporidiosis. Also do we need to consider combination therapy 
Citation: Sajjad A, Sunil K (2015) Treatment Outcomes with Nitazoxanide in Immunocompetent Adults Naive Patients with Cryptosporidiosis; Do We Need Combination Therapy with Paromomycin or Azithromycin?. Trop Med Surg 3: 198. doi:10.4172/2329-9088.1000198

Page 2 of 4

which includes nitazoxanide with paromomycin or azithromycin particularly in patient with high relapse or recurrence of diarrhea?

\section{Study Design}

\section{Study population}

This prospective study was conducted at Sindh Institute of Urology and Transplantation (SIUT) in Karachi from February to September 2012. We included all immunocompetent adult patients above the age of 16 years who have been diagnosed with cryptosporidiosis on stool microscopy.

We excluded patients if they had any immunodeficiency such as HIV infection, hematological malignancy, solid organ transplantation or receiving steroids or other immunosuppressive drugs. We also did not enrolled patients previously diagnosed with cryptosporidiosis and had taken nitazoxanide, paromomycin or azithromycin in last 4 weeks prior their diagnosis.

\section{Study design}

Patients who were diagnosed with cryptosporidiosis where enrolled in this study after their written informed consent. The data collection sheet was filled. We prescribed nitazoxanide $500 \mathrm{mg}$ twice daily for 7 days. Although 3 days of therapy is recommended in immunocompetent adults, we chose a longer treatment period based on our clinical experience over the years, high recurrence/relapse with 3 days of Nitazoxanide. At follow-up visit after 1 week treatment with nitazoxanide clinical response was documented. At 6 weeks patients were called on phone to assess whether diarrhea was ongoing, resolved or had relapsed during the past 6 weeks.

\section{Results}

A total of 58 patients who had cryptosporidiosis were seen in the gastroenterology clinic during study period. There were 31 (53.4\%) males and $27(46.6 \%)$ females. The mean age was 33.4 years with standard deviation \pm 9.2 . No statistical significance was seen in clinical presentation of cryptosporidiosis in both male and female genders (Table 1).

\begin{tabular}{|c|c|c|c|c|}
\hline & & Male $n=31$ & Female $n=27$ & P-Value \\
\hline \multirow{2}{*}{$\begin{array}{l}\text { Duration of illness } \\
\text { (days) }\end{array}$} & $<=5$ & $24(77.4 \%)$ & $19(44.2 \%)$ & \multirow{2}{*}{0.54} \\
\hline & $>5$ & $7(22.6 \%)$ & $8(53.3 \%)$ & \\
\hline \multirow[t]{2}{*}{ Episodes of Diarrhea } & $\begin{array}{l}\text { 1st } \\
\text { Episode }\end{array}$ & $5(16.1 \%)$ & $11(40.7 \%)$ & \multirow{2}{*}{0.85} \\
\hline & $\begin{array}{l}\text { Recurrent } \\
\text { episodes }\end{array}$ & $26(83.9 \%)$ & $16(59.2 \%$ & \\
\hline \multirow{2}{*}{$\begin{array}{l}\text { Stool Frequency per } \\
\text { day }\end{array}$} & $<=5$ & $9(29.0 \%)$ & $13(59.1 \%)$ & \multirow{2}{*}{0.13} \\
\hline & $>5$ & $22(60.3 \%)$ & $14(51.9 \%)$ & \\
\hline \multirow[t]{2}{*}{ Stool Consistency } & Watery & $28(90.3 \%)$ & $22(81.5 \%)$ & \multirow{2}{*}{0.33} \\
\hline & Formed & $3(9.7 \%)$ & $5(18.5 \%)$ & \\
\hline \multirow[t]{2}{*}{ Blood in Stool } & Yes & $1(3.2 \%)$ & $2(7.4 \%)$ & \multirow{2}{*}{0.47} \\
\hline & No & $30(96.8 \%)$ & $25(92.6 \%)$ & \\
\hline
\end{tabular}

\begin{tabular}{|c|c|c|c|c|}
\hline \multirow[t]{2}{*}{ Mucus in Stool } & Yes & 2 (6.5\% & $6(22.2 \%)$ & \multirow{2}{*}{0.08} \\
\hline & No & 29 (93.5\%) & $21(77.8 \%)$ & \\
\hline \multirow[t]{2}{*}{ Abdominal Pain } & Yes & $28(90.3 \%)$ & $26(26 \%)$ & \multirow{2}{*}{0.37} \\
\hline & No & $3(9.7 \%)$ & $1(3.7 \%)$ & \\
\hline \multirow[t]{2}{*}{ Vomiting } & Yes & $15(48.4 \%)$ & 15 (55.6\%) & \multirow{2}{*}{0.58} \\
\hline & No & $16(51.6 \%)$ & $12(44.4 \%)$ & \\
\hline \multirow[t]{2}{*}{ Weight Loss } & Yes & $6(19.5 \%)$ & $11(40.7 \%)$ & \multirow{2}{*}{0.07} \\
\hline & No & 25 (80.6\%) & $16(59.3 \%)$ & \\
\hline \multirow[t]{2}{*}{ Fever during Diarrhea } & Yes & $24(77.4 \%)$ & $18(66.6 \%)$ & \multirow{2}{*}{0.36} \\
\hline & No & $7(22.6 \%)$ & 9 (33.3\%) & \\
\hline \multirow[t]{2}{*}{ Dehydration } & Yes & $29(93.5 \%)$ & $21(77.8 \%)$ & \multirow{2}{*}{0.08} \\
\hline & No & $2(6.5 \%)$ & $6(22.2 \%)$ & \\
\hline \multirow[t]{2}{*}{ Headache } & Yes & $7(22.6 \%)$ & $12(44.4 \%)$ & \multirow{2}{*}{0.07} \\
\hline & No & $24(77.4 \%)$ & $15(55.6 \%)$ & \\
\hline \multirow[t]{2}{*}{ Fatigue } & Yes & $29(93.5 \%)$ & $27(48.2 \%)$ & \multirow{2}{*}{0.17} \\
\hline & No & $2(6.5 \%)$ & $0(0 \%)$ & \\
\hline \multirow[t]{2}{*}{ Joint Pain } & Yes & $12(38.7 \%)$ & $13(48.1 \%)$ & \multirow{2}{*}{0.46} \\
\hline & No & $19(61.3 \%)$ & $14(51.9 \%)$ & \\
\hline
\end{tabular}

Table 1: Clinical Presentation of Cryptosporidiosis in Male and Female Patients.

Out of 58 patients 43 patients had duration of illness consist of 2 to 4 days (Figure 1). While most of patients had stool frequency greater than 6 episodes per day (Figure 2).

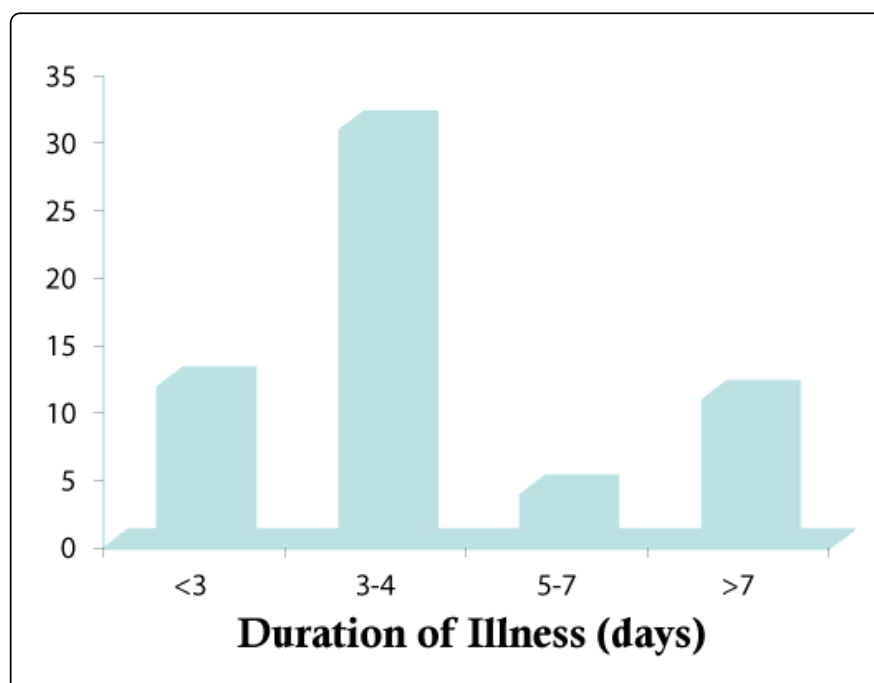

Figure 1: Duration of Diarrheal Illness in Immunocompetent Adult Cryptosporidiosis Patients. 


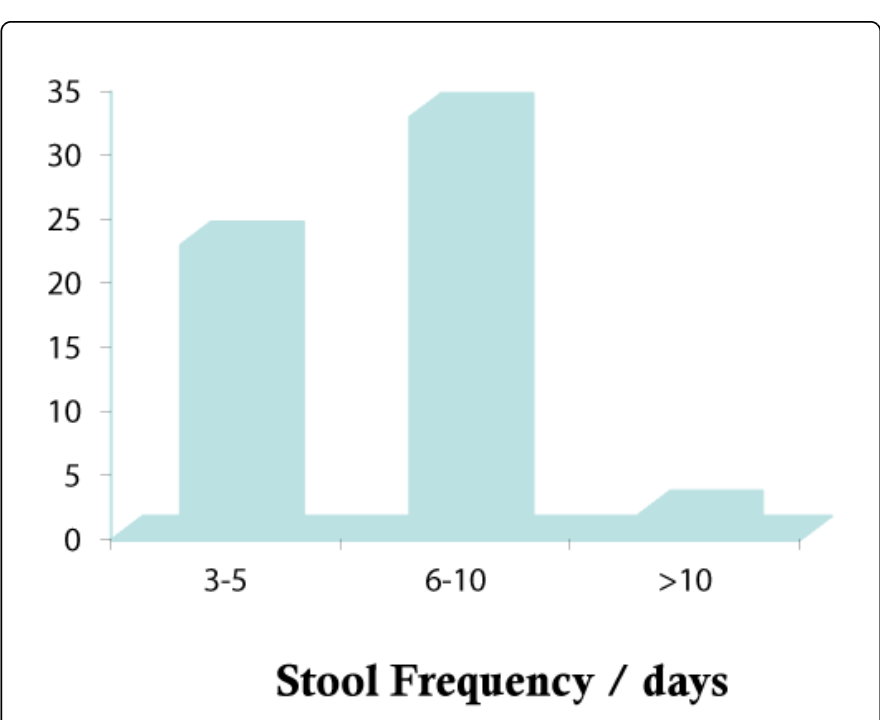

Figure 2: Stool Frequencies in Immunocompetent Adult Cryptosporidiosis Patients.

\section{Nitazoxanide treatment outcome in immunocompetent adult cryptosporidiosis patients}

58 patients were diagnosed with cryptosporidiosis by stool microscopy and were treated with nitazoxanide $500 \mathrm{mg}$ twice a day for 7 days. All 58 (100\%) reported resolution of diarrhea after 7 days of nitazoxanide treatment. At 6 week follow up one patient could not be contacted so lost to follow up. However, at 6 weeks follow up by phone, $40(70.1 \%)$ patients reported recurrence of diarrhea whereas only 17 $(29.9 \%)$ reported resolution of diarrhea. Nitazoxanide was well tolerated by most of patients and no adverse event being reported. Side effects reported include nausea, vomiting, epigastric discomfort or pain and rarely headache.

\section{Discussion}

Cryptosporidiosis is emerging threat to world population. It could be because of difficulties in eradication and killing of pathogens by routine methods of water purification like chlorination, widely used in world [8]. Cryptosporidium is chlorine resistant. Its Prevalence is increasing day by day $[9,10]$. There have been quite number of Cryptosporidiosis outbreaks being reported after the massive outbreak of Milwaukee in 1993, affected estimated 403,000 people [11].

Nitazoxanide is novel drug for the treatment of Cryptosporidiosis and Giardiasis. Nitazoxanide as an antiparasitic is one of the drug which has recently been discovered in last few decades.

To the best of our knowledge, this is the first study conducted on nitazoxanide treatment outcomes in cryptosporidiosis immunocompetent adult patients presenting with acute diarrhea in Pakistan.

Nitazoxanide is licensed for treatment of cryptosporidiosis and giardiasis and have been approved by FDA. Nitazoxanide is new thiazolide antiparasitic agent that shows excellent in vitro activity against a wide variety of protozoa and helminthes [12]. There is no documented drug-drug interactions reported till yet showing very good safety profile of nitazoxanide. Other enteric protozoan infections caused by Giardia intestinalis, Entamoeba histolytica, Blastocystis hominis and Isospora belli can also be treated with nitazoxanide [13]. Some studies have shown efficacy of nitazoxanide against Clostridium difficile colitis [14]. Nitazoxanide has also been used in the treatment of severe rotavirus diarrhea [15].

Guidelines recommend a 3-day course of nitazoxanide, $500 \mathrm{mg}$ twice daily in adults [5]. Although we gave 7 days of therapy because of persistence of diarrhea commonly seen in our outpatients department with 3 days course and we obtained satisfactory treatment response in the short term with resolution of diarrhea in all 58 patients after 7 days of treatment with nitazoxanide. But in long term, patients reported a high recurrence rate with recurrence of diarrhea in 6 weeks period time.

There may be need of combination therapy in immunocompetent adult patients with cryptosporidiosis particularly in patients with high recurrence/relapse. This includes nitazoxanide with paromomycin or azithromycin. Nitazoxanide has ability to inhibit the growth of sporozoites of $C$. parvum on its own, but when used in combination with azithromycin and rifampin, shown synergistic in vitro activity with both in suppressing growth of $C$. parvum by $83.9 \%$ and $79.8 \%$, respectively, compared with $56.1 \%$ when used alone [16]. Paromomycin and/or azithromycin in combination with nitazoxanide have been tested in double blind randomized trials for the treatment of cryptosporidiosis in immunocomprised patients such as those with HIV/AIDS, and the results found to be encouraging $[13,17,18]$. But combination therapy has not being well tested in immunocompetent adult patients particularly in high relapse or recurrence individual. So further studies need to be conducted regarding optimal duration, dosing and antimicrobial combinations with paramomycin or azithromycin in the treatment of cryptosporidiosis in immunocompetent adult patients with high recurrence.

\section{Conclusion}

Nitazoxanide is a new nitrothiazole compound with broadspectrum activity against numerous intestinal protozoa, helminths, and anaerobic bacteria and have very good bio-safety profile. Nitazoxanide is FDA approved for cryptosporidiosis and giardiasis. Guidelines recommend a 3-day course of nitazoxanide, $500 \mathrm{mg}$ twice daily in adults [5]. Although we gave 7 days of therapy because of high relapse/ recurrence rate in our patients and we obtained satisfactory treatment response in the short term with resolution of diarrhea in all 58 patients after 7 days of treatment with nitazoxanide. But in long term patients reported a high recurrence rate with recurrence of diarrhea in 6 weeks period time.

There is need of combination therapy which includes nitazoxanide with paromomycin or azithromycin in high recurrence/relapse immunocompetent adult patients with cryptosporidiosis.

Further randomized controlled trails are needed regarding optimal duration of nitazoxanide and combination therapy with paromomycin or azithromycin.

\section{References}

1. Hunter PR, Hughes S, Woodhouse S, Syed Q, Verlander NQ, et al. (2004) Sporadic cryptosporidiosis case-control study with genotyping. Emerg Infect Dis 10: 1241-1249.

2. Chen XM, Keithly JS, Paya CV, LaRusso NF (2002) Cryptosporidiosis. N Engl J Med 346: 1723-1731. 
Citation: Sajjad A, Sunil K (2015) Treatment Outcomes with Nitazoxanide in Immunocompetent Adults Naive Patients with Cryptosporidiosis; Do We Need Combination Therapy with Paromomycin or Azithromycin?. Trop Med Surg 3: 198. doi:10.4172/2329-9088.1000198

Page 4 of 4

3. Robertson B, Sinclair MI, Forbes AB, Veitch M, Kirk M, et al. (2002) Case-control studies of sporadic cryptosporidiosis in Melbourne and Adelaide, Australia. Epidemiol Infect 128: 419-431.

4. Shirley DA, Moonah SN, Kotloff KL (2012) Burden of disease from cryptosporidiosis. Curr Opin Infect Dis 25: 555-563.

5. Pantenburg B, Cabada MM, White AC Jr (2009) Treatment of cryptosporidiosis. Expert Rev Anti Infect Ther 7: 385-391.

6. Rossignol JF, Ayoub A, Ayers MS (2001) Treatment of diarrhea caused by Giardia intestinalis and Entamoeba histolytica or E. dispar: a randomized, double-blind, placebo-controlled study of nitazoxanide. J Infect Dis 184: 381-384.

7. Rossignol JF, Lopez-Chegne N, Julcamoro LM, Carrion ME, Bardin MC (2012) Nitazoxanide for the empiric treatment of pediatric infectious diarrhea. Trans R Soc Trop Med Hyg 106: 167-173.

8. Shields JM, Hill VR, Arrowood MJ, Beach MJ (2008) Inactivation of Cryptosporidium parvum under chlorinated recreational water conditions. J Water Health 6: 513-520.

9. Yoder JS, Harral C, Beach MJ; Centers for Disease Control and Prevention (CDC) (2010) Cryptosporidiosis surveillance - United States, 2006-2008. MMWR Surveill Summ 59: 1-14.

10. Yoder JS, Wallace RM, Collier SA, Beach MJ, Hlavsa MC; Centers for Disease Control and Prevention (CDC) (2012) Cryptosporidiosis surveillance--United States, 2009-2010. MMWR Surveill Summ 61: 1-12.
11. Morris RD, Naumova EN, Griffiths JK (1998) Did Milwaukee experience waterborne cryptosporidiosis before the large documented outbreak in 1993? Epidemiology 9: 264-70.

12. Rossignol JF, Ayoub A, Ayers MS (2001) Treatment of diarrhea caused by Cryptosporidium parvum: a prospective randomized, double-blind, placebo-controlled study of Nitazoxanide. J Infect Dis 184: 103-6.

13. Rossignol JF (2010) Cryptosporidium and Giardia: treatment options and prospects for new drugs. Exp Parasitol 124: 45-53.

14. Musher DM, Logan N, Bressler AM, Johnson DP, Rossignol JF (2009) Nitazoxanide versus vancomycin in Clostridium difficile infection: a randomized, double-blind study. Clin Infect Dis 48: e41-46.

15. Rossignol JF, El-Gohary YM (2006) Nitazoxanide in the treatment of viral gastroenteritis: a randomized double-blind placebo-controlled clinical trial. Aliment Pharmacol Ther 24: 1423-30.

16. Giacometti A, Cirioni O, Barchiesi F, Ancarani F, Scalise G (2000) Activity of nitazoxanide alone and in combination with azithromycin and rifabutin against Cryptosporidium parvum in cell culture. J Antimicrob Chemother 45: 453-456.

17. Thielman NM, Guerrant RL (2004) Clinical practice. Acute infectious diarrhea. N Engl J Med 350: 38-47.

18. Allam AF, Shehab AY (2002) Efficacy of azithromycin, praziquantel and mirazid in treatment of cryptosporidiosis in school children. J Egypt Soc Parasitol 32: 969-978. 